\title{
Improving Renoprotective Therapy by Targeting the Body Sodium Balance
}

\author{
Liffert Vogt $^{\mathrm{a}}$ Ron T. Gansevoort ${ }^{\mathrm{b}}$ \\ aDepartment of Internal Medicine, Section Nephrology, Amsterdam Cardiovascular Sciences, Amsterdam UMC, \\ University of Amsterdam, Amsterdam, The Netherlands; ${ }^{\text {b}}$ Division of Nephrology, University Medical Center \\ Groningen, University of Groningen, Groningen, The Netherlands
}

Owing to both their blood pressure (BP)-lowering and antiproteinuric effects, renin-angiotensin-aldosterone system (RAAS) inhibitors are generally regarded as the pharmacological cornerstone of renoprotective therapy in chronic kidney disease (CKD). Although RAAS inhibitors effectively control $\mathrm{BP}$ and reduce proteinuria in most patients, large interindividual differences in treatment effect exist, presumably explaining why some patients still progress to kidney failure.

To optimize RAAS inhibitor renoprotective action, various strategies have been studied in the past 2 decades, including additional treatment with diuretics and dietary measures $[1,2]$. These studies demonstrate that the efficacy of single RAAS inhibition, either by an angiotensinconverting enzyme inhibitor or an angiotensin II type 1 receptor blocker, can be significantly improved in diabetic as well as nondiabetic CKD by adding a thiazide diuretic, restricting daily sodium consumption, or a combination of both measures $[1,2]$. Also, patients with an inadequate response to single RAAS blockade in terms of $\mathrm{BP}$ or proteinuria reduction catch up once one of these measures has been added [2]. These interventions are therefore crucial for eliminating variability in treatment efficacy.

Data showing that modulation of the sodium balance may also result in improved long-term outcomes are derived from the most recently conducted large randomized controlled clinical trials in the nephrology field, including, respectively, the addition of sodium-glucose cotrans-

karger@karger.com

(c) 2021 S. Karger AG, Basel

www.karger.com/ajn

Karger! porter-2 (SGLT2) inhibitors and the novel mineralocorticoid receptor antagonist finerenone to patients treated with single RAAS blockade [3, 4]. Besides potentially other beneficial effects, both drug classes stimulate natriuresis and have shown to reduce both $\mathrm{BP}$ and proteinuria while given on top of the maximum tolerated RAAS blockade [3, 4].

The recent DAPA-CKD and FIDELIO-DKD studies confirm that these agents slow down the progression of kidney disease and lower the incidence of clinically relevant renal and cardiovascular events - again against a background of RAAS blockade $[4,5]$. Whether long-term cardio and renoprotection by SGLT2 inhibitors and finerenone are solely obtained owing to their natriuresisstimulating properties or that other effects are also important needs to be determined, but the considerations emphasize the crucial - yet impeding - role of increased sodium retention in the efficacy of CKD treatment.

The reason why proteinuria patients are particularly sensitive to increased sodium retention and subsequent extracellular fluid volume expansion was not well understood for a long time. Still, recent experimental data indicate that proteinuria causes upregulation of epithelial sodium channel $(\mathrm{ENaC})$ via glomerular plasminogen filtration along with other plasma proteins and subsequent conversion of plasminogen to plasmin [6]. Plasmin, a serine protease, enhances $\mathrm{ENaC}$ activity by cleaving the extracellular loop of the $\mathrm{ENaC} \gamma$ subunit. These sequelae indicate that proteinuria directly leads to increased so- 
dium reabsorption in the collecting tubule [6]. One might therefore hypothesize that proteinuric patients particularly benefit from diuretics that target $\mathrm{ENaC}$ [6].

In this issue, Shen et al. [7] touch upon this point - yet with another hypothesis - by studying the antiproteinuric effects of 8-week amiloride $5 \mathrm{mg}$ b.i.d. and 8-week triamterene $50 \mathrm{mg}$ b.i.d., respectively, on top of the maximum tolerable dose of a single RAAS blocker in an open-label randomized cross-over study, including 12 proteinuric patients (mean $2.9 \mathrm{~g}$ /day) caused by various etiologies (including diabetes) with a relatively preserved kidney function (mean eGFR $81 \mathrm{~mL} / \mathrm{min} / 1.73 \mathrm{~m}^{2}$ ) and reasonably controlled BP (137/75 mm Hg) [7]. Because previous animal research indicated that amiloride attenuates proteinuria and glomerulosclerosis independent of $\mathrm{ENaC}$ inhibition, the authors hypothesized that amiloride, but not triamterene, would have an additional antiproteinuric effect on top of a single RAAS blockade. Their study, however, could not demonstrate a differential response concerning change in proteinuria ( 39 vs. $33 \% 24$-h proteinuria reduction for amiloride and triamterene, respectively). Distinct effects concerning BP and eGFR were, however, observed after both treatments.

Amiloride reduced mean systolic BP by $12 \mathrm{~mm} \mathrm{Hg}$ without affecting eGFR. In contrast, triamterene did not affect $\mathrm{BP}$, while mean eGFR dropped with $9 \mathrm{~mL} / \mathrm{min} / 1.73$ $\mathrm{m}^{2}-$ an effect that disappeared upon withdrawal of the drug after 8 weeks. Unfortunately, the study could not substantiate why these distinct changes occurred. Assessment of natriuretic efficacy, measured GFR and renal plasma flow, plasma and urinary RAAS hormones, and dietary status regarding daily sodium and protein consumption would have been helpful here.

The authors conclude that proteinuria reduction by amiloride and triamterene was independent of RAAS blockade. It is questionable, however, whether this is correct. Because all study participants were on RAAS inhibition throughout the study, and the authors could not formally test the intrinsic antiproteinuric effect of both drugs. Furthermore, an alternative explanation seems more likely. Provided that the reduction in proteinuria after establishing a negative sodium balance coincides with an initial GFR drop, and the proteinuria effect is usually attributed to a decrease in intraglomerular pressure caused by reversing glomerular hyperfiltration, that is, the critical pathophysiological feature in CKD [1-3].

This phenomenon might also have been responsible for the proteinuria reduction observed after selective inhibition of renal $\mathrm{ENaC}$ with triamterene. However, amiloride inhibits not only renal $\mathrm{ENaC}$ but also nonrenal
$\mathrm{ENaC}$ as present in the vasculature, intestines, and central nervous system and blocks other ion channels as well [8]. These latter actions may have been responsible for the additional systemic BP decrease as observed by Shen et al. [7]. In theory, distinct tubular handling of potassium by both agents may also differentially affect $\mathrm{BP}$ and proteinuria [8]. Still, regarding the similar changes in plasma potassium, this seems not to have occurred.

Regardless of the exact mechanisms explaining proteinuria reduction, 2 other questions remain. Firstly, it is unknown whether the comparable antiproteinuric efficacy of both potassium-sparing agents will translate into equal cardio and renal protection in the long term. Based on the observation that amiloride further improves BP control indicates that the odds seem to benefit amiloride. Secondly, aldosterone antagonists also influence $\mathrm{ENaC}$ function via blockade of the mineralocorticoid receptor. In diabetic kidney disease, finerenone at the 15 and $20 \mathrm{mg}$ daily dose on top of maximum tolerable dosed RAAS blockade reduced proteinuria with 33 and $38 \%$, respectively, as compared to placebo. These effect sizes may not substantially differ from the introduction of amiloride or triamterene. It is, therefore, an interesting question whether the protective effect of finerenone in DKD patients on kidney outcomes in the FIDELIO study [4] would have been similar with the use of amiloride or triamterene - drugs that are widely available at low costs since their introduction in the 60s of the last century.

What can we learn from Shen et al. [7] study and evidence from the above outlined short-term and long-term outcome studies in diabetic and nondiabetic CKD? Undoubtedly, all studies confirm that reaching an adequate body sodium status represents an important treatment goal by itself in CKD treatment. Dietary sodium restriction, thiazide diuretics, SGLT2 inhibition, aldosterone antagonists, and the potassium-sparing diuretics amiloride and triamterene lead to a reduction in proteinuria when given on top of a single RAAS blockade; however, mechanisms by which this additional proteinuria reduction will be obtained might be different for each approach, and some of the decrease in proteinuria might even occur beyond their sodium-modulating effects. The jury is still out whether these differences will also be relevant to prevent new end-stage renal disease cases.

\section{Conflict of Interest Statement}

L.V. received consultancy fees from Astra-Zeneca and Bayer. R.G. received grants and consultancy fees from Astra-Zeneca, Bayer, and Mundi Pharma. 


\section{References}

1 Kwakernaak AJ, Krikken JA, Binnenmars SH, Visser FW, Hemmelder MH, Woittiez AJ, et al. Effects of sodium restriction and hydrochlorothiazide on RAAS blockade efficacy in diabetic nephropathy: a randomised clinical trial. Lancet Diabetes Endocrinol. 2014;2(5): 385-95.

2 Vogt L, Waanders F, Boomsma F, de Zeeuw D, Navis G. Effects of dietary sodium and hydrochlorothiazide on the antiproteinuric efficacy of losartan. J Am Soc Nephrol. 2008; 19(5):999-1007.
3 Cherney D, Lund SS, Perkins BA, Groop PH, Cooper ME, Kaspers S, et al. The effect of sodium glucose cotransporter 2 inhibition with empagliflozin on microalbuminuria and macroalbuminuria in patients with type $2 \mathrm{dia}-$ betes. Diabetologia. 2016;59(9):1860-70.

4 Bakris GL, Agarwal R, Anker SD, Pitt B, Ruilope LM, Rossing P, et al. Effect of finerenone on chronic kidney disease outcomes in type 2 diabetes. N Engl J Med. 2020;383(23): 2219-29.

5 Heerspink HJL, Stefánsson BV, Correa-Rotter R, Chertow GM, Greene T, Hou FF, et al. Dapagliflozin in patients with chronic kidney disease. N Engl J Med. 2020;383(15):1436-46.
6 Hinrichs GR, Jensen BL, Svenningsen P. Mechanisms of sodium retention in nephrotic syndrome. Curr Opin Nephrol Hypertens. 2020;29(2):207-12.

7 Shen W, Alshehri MA, Desale S, Wilcox CS The effect of amiloride on proteinuria in patients with proteinuric kidney disease. Am J Nephrol. 2021.

8 Teiwes J, Toto RD. Epithelial sodium channel inhibition in cardiovascular disease. A potential role for amiloride. Am J Hypertens. 2007; 20(1):109-17. 